\title{
PROPUESTA DE RESTRUCTURACIÓN DEL FONDO DE PUBLICACIONES DE LA U.D.C.A
}

\section{PROPOSAL FOR THE RESTRUCTURATION OF U.D.C.A'S PUBLICATION FOUNDATION}

\author{
Norella Castro Rojas ${ }^{1}$ \\ María Andrea Contreras Nieto ${ }^{2}$
}

\section{RESUMEN}

El papel que han llevado a cabo durante años las editoriales universitarias en el mundo refleja no solamente una unidad indispensable en las instituciones de educación superior sino que contribuyen ampliamente a la expansión del conocimiento y del saber. Sin embargo, su reto y su labor cada vez se hacen más inciertos, ya que el rol del libro en el sistema educativo universitario es calificado como pobre, debido a las posiciones que algunos docentes mantienen con relación al mismo. Actualmente, ni en los hogares, ni en los colegios, ni en las universidades, se crea un gusto o deleite por la lectura, generadora y creadora de ideas, pero sí inculcan la creencia que los medios y el encantamiento generado por la Internet son los más adecuados, sin saber que son los factores que amenazan con relegar el libro del mundo del intelecto. Todo lo anterior, conlleva a que las editoriales universitarias generen estrategias de mercadeo en sus ediciones, con el fin de evitar la reprografía ilegal, la piratería y la acumulación de stock de ejemplares en sus bodegas.

Palabras clave: Editoriales universitarias, comercialización, reprografía ilegal, cultura impresa.

\footnotetext{
${ }^{1}$ Comunicadora Social y Periodista, Coordinadora del Fondo de Publicaciones de la Universidad de Ciencias Aplicadas y Ambientales U.D.C.A. norellac@udca.edu.co.

${ }^{2}$ Médica Veterinaria, Maestría en Dirección Universitaria. EdD Administración en Educación Superior. Coordinadora Centro de Gestión del Conocimiento y la Innovación de la Universidad del Rosario.maria.contreras58@urosario.edu.co.
}

The role accomplished worldwide during years by university editorials not only reflects an essential unity within the institution of higher education, but also contributes amply to the expansion of knowledge and understanding. However, its challenge and task each time is getting more uncertain, since the role of the book within the universities educational system is rated as poor, due to the positions related to it, sustained by some professors. At present, neither at home, nor at school or university, a pleasure or delight for reading, the generator and the creator of ideas, is fomented, but the belief, that the media and the enchantment generated by the internet are the most adequate ones is inculcated, without knowing that these are the factors that threaten to relegate the book from the world of intellect. All this together aids the university editorials to generate market strategies with their editions, in order to avoid the illegal reprography, the piracy and the accumulation of a stock of copies in their storerooms.

Key words: University editorials, commercialization, illegal reprography, printed culture.

\section{INTRODUCCIÓN}

Las instituciones de educación superior son consideradas como uno de los principales espacios donde se genera el conocimiento y donde se promueve el desarrollo intelectual, herramienta pedagógica en el proceso de enseñanza-aprendizaje y que puede ser socializada a través de las editoriales universitarias. Esta actividad es un quehacer académico que va ligado a la vida 
universitaria, a la cual se le reconocen los siguientes objetivos: servir de apoyo a la docencia, divulgar la producción intelectual, la cultura y los resultados de la investigación y estrechar los vínculos de la universidad con la sociedad (Polo, 2005).

Para llevar a cabo estas gestiones, las editoriales universitarias deben velar por una calidad técnica, formal y de contenido independiente del tipo de soporte que se utilice en su edición, ya sea en papel impreso o en medio digital. Se tiene la creencia que con el desarrollo tecnológico y la nueva era digital, los libros impresos tienden a desaparecer; sin embargo, la cultura digital y la cultura impresa no son opuestas sino que coexisten. Esto hace que el rol de las editoriales universitarias cambie para que pueda dar respuesta a los nuevos retos. La U.D.C.A, no ha sido ajena a este proceso y es por ello que se elabora esta propuesta que le permita al Fondo de Publicaciones ofrecer novedosos esquemas y políticas y enfrentar estos desafíos y responsabilidades.

\section{DESARROLLO HISTÓRICO DEL LIBRO Y LAS EDITORIALES UNIVERSITARIAS}

La evolución del libro ha sido paralela al desarrollo histórico de la universidad. Los talleres de copistas y los códices manuscritos elaborados en los monasterios y catedrales surgen como necesidad de proveer libros a la comunidad universitaria. Una de las primeras formas de libro, se desarrolló entre el siglo II y el IV a través del códices, formado por hojas plegadas, unidas y encuadernadas que tenía una estructura similar a nuestro libro actual. Este formato reemplazó al rollo, el cual requería del uso de las dos manos para su lectura. Estos recursos coexistieron durante varios años (Furió, 2005). Existen métodos más antiguos, especialmente en las civilizaciones mesopotámicas, a través de planchas de barro, que data unos 4.000 años a. de C., sin olvidar el uso de las tablillas de arcilla y del papiro, propio de los griegos, romanos y egipcios. La impresión cambió y la forma de presentación del libro también, a partir del siglo VI a. de C., cuando los chinos manipulaban inteligentemente los bloques de madera con caracteres incisos y, posteriormente, la impresión a partir de bloques móviles. Finalmente, la revolución del libro se logró a partir del año 1456, con Gutenberg, a quien se le atribuye la invención de la imprenta de tipos móviles (Cardona et al. 2007).
El libro ha jugado un papel importante en el desarrollo cultural de la sociedad, pero más en la universidad, como medio de consulta, difusión de ideas y de hallazgos científicos. Las universidades creadas en el medievo, como París, Bolonia, Salerno, Oxford y Salamanca y otras, fueron las impulsoras del auge del libro y de la cultura escrita, pues los estudiantes los requerían no sólo para profundizar las cátedras impartidas por los maestros, sino también por la necesidad de leer directamente autores clásicos y modernos. Derivado de ello tuvieron que buscar la manera de poseer varios títulos y diversos ejemplares de una misma obra. Así empieza el desarrollo de las editoriales, donde la universidad pasa de ser una organización transmisora de saberes a generadora de conocimiento. Dicho concepto fue introducido por la Universidad Alemana, con el modelo de Humboldt, quien incluyó la ciencia y la investigación dentro de las funciones sustantivas, extendiéndose a las otras instituciones de educación superior, a nivel mundial. Durante el siglo XX continúa el desarrollo de estas editoriales, debido a la gran expansión del sistema educativo profesional, en donde se incrementa el número de instituciones el número estudiantes matriculados (Furió, 2005).

En Estados Unidos, la primera universidad en utilizar el término de University Press fue Cornell, en 1869, seguida de Pensilvania y de John Hopkins. El resto de editoriales fueron establecidas después de 1935 y agrupadas, actualmente, por la Association of American University Press. En América Latina, la creación de universidades se ha multiplicado en las últimas décadas lo que ha hecho que las editoriales universitarias se conviertan poco a poco, en emporios empresariales del libro, como la Editorial de la Universidad del Rosario (Argentina), la editorial de la UNAM (México) y la Universidad Nacional (Colombia) (Cardona et al. 2007).

\section{CARACTERÍSTICAS GENERALES DE LAS EDITORIALES UNIVERSITARIAS}

Hablar de editoriales universitarias es referirse actualmente a organizaciones que actúan, independientemente, dentro del ámbito de una universidad, editando y gerenciando la industria cultural. Algunas editoriales, específicamente las calificadas como emporios (casos colombianos Editorial Universidad de Antioquia, Editorial Pontificia Universidad Javeriana, Editorial Universidad Externado de Colombia y otras más), han aportado 
significativamente al desarrollo y creación de otros Fondos Editoriales, adoptando esquemas, políticas y características similares para su evolución y su sostenimiento (Cardona et al. 2007). Son peculiaridades generales y propias, implementadas por la mayoría de las editoriales universitarias, las cuales se pueden resumir en gestión administrativa, edición y producción, mercadeo y comercialización (Tabla 1).

Una vez analizadas y discutidas estas características en diferentes foros y congresos, especialmente en el VI Congreso Internacional de Editores Universitarios de América Latina y el Caribe, el cual se realizó en la ciudad de San José de Costa Rica, del 22 al 24 de agosto de 2007, se concluye que las editoriales universitarias, frente a este mundo en permanente proceso de cambio, se deben fortalecer y buscar alternativas, como diversidad cultural, afianzar las relaciones entre autor-editorcomercializador y asegurar la calidad editorial, para su permanencia y su visibilidad, sin dejar de lado la enorme responsabilidad social. Es claro lo que resalta De Sagastizábal (2005) respecto que la actividad editorial universitaria se debe esforzar porque sus criterios y políticas estén en concordancia con las necesidades y los objetivos de la universidad, a la cual pertenece, sin olvidar y dejar de lado el desarrollo del país, en sus aspectos científicos, tecnológicos, humanísticos, sociales y culturales.

Sierra (2005), Sicard (2005) y Cardona et al. (2007) plantean y discuten la importancia de afianzar el propósito de elevar el nivel de conocimiento de los estudiantes que ingresan a las universidades y los que están actualmente en ellas, a través de la corrección de deficiencias dejadas por el vacío existente en cuanto a textos adecuados a nivel de educación superior, pues la mayoría son producto de traducciones o editados en otros idiomas.

Es importante aclarar que en el mundo de la edición, las diferencias entre una editorial universitaria y una comercial son significativas de fondo mas no de forma, pues las primeras, cuyo objetivo principal es su desempeño social, tiene definida tres misiones específicas: la docencia, la investigación y la difusión cultural. Por su parte, las comerciales, tal como lo afirma Sierra (2005), aunque se encuentran inmersas en la misma sociedad, no tiene los compromisos sociales, respondiendo únicamente a exigencias de lucro y publicando obras o revistas independientemente de si éstas constituyen legítimos aportes al saber o no.

\section{RETOS DE LAS EDITORIALES, TAMBIÉN PARA EL CASO U.D.C.A}

Varios autores, como Polo (2005), Hernández (2005) y Cavaliere (2005), coinciden que la producción editorial de las universidades debe enfrentar nuevos desafíos, cada vez más exigentes, académica y socialmente, en el cumplimiento ético de la calidad pedagógica y científica, retos que también persigue y establece la Universidad. Como su objetivo es servir de instrumento de extensión del conocimiento y del saber, debe irrumpir en los recientes y estrepitosos cambios tecnológicos y comerciales para su progreso, así:

- Lograr una gestión editorial profesional que haga que las editoriales puedan superar su actitud pasiva, estableciendo con claridad la finalidad de la editorial, dándole sentido, continuidad y consistencia a sus catálogos universitarios.

- Diseñar políticas editoriales coherentes de acuerdo con el proyecto educativo institucional y con la misión de cada universidad, permitiendo definir planes de acción editoriales y comerciales, así como procesos de evaluación.

- Impulsar proyectos vinculados a propósitos y planes comunes para el establecimiento de asociaciones y de redes para edición y distribución.

- Concebir proyectos universitarios de acuerdo con los lectores a los que se espera ofrecer las ediciones de la editorial.

- Ofrecer mejores condiciones contractuales y promocionales a docentes e investigadores, tanto de las universidades como de institutos de investigación, quienes constituyen el capital intelectual.

- Cambiar las estrategias de las editoriales universitarias, es decir, perpetrar en ediciones con autores diferentes a los docentes universitarios, publicando traducciones, editando coediciones, comprando derechos de autor extranjeros.

- Establecer otros productos editoriales, diferentes al libro impreso, que hagan uso de nuevos soportes y formatos que permitan la difusión y la divulgación del conocimiento, ejemplo de ello, los libros virtuales. 


\begin{tabular}{|c|c|c|c|}
\hline \multirow{2}{*}{ 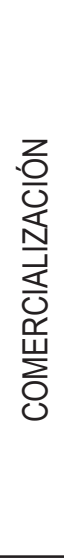 } & $\begin{array}{l}\stackrel{O}{Z} \\
\text { 离 } \\
\text { 㞱 }\end{array}$ & 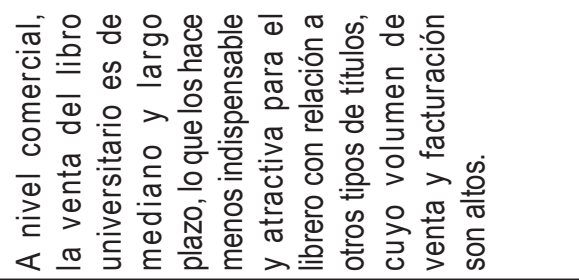 & 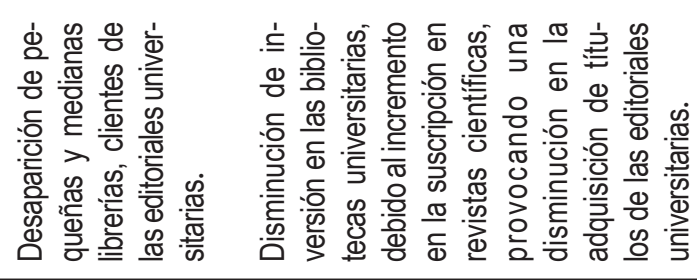 \\
\hline & 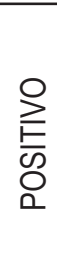 & 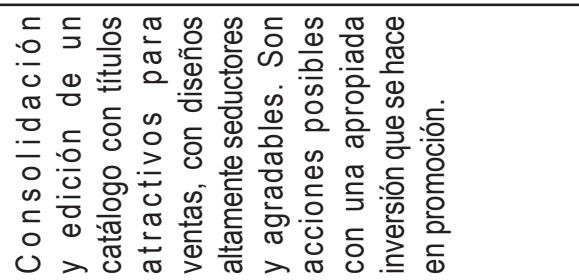 & 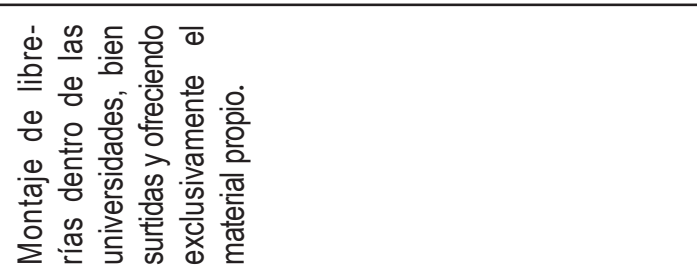 \\
\hline \multirow{2}{*}{ 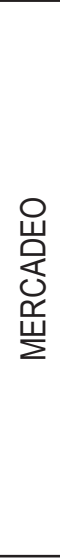 } & $\begin{array}{l}\stackrel{O}{Z} \\
\text { 悤 } \\
\text { 㞱 }\end{array}$ & 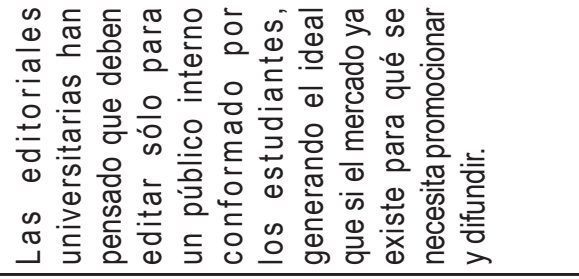 & 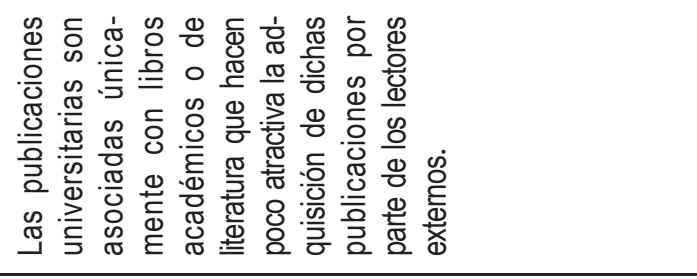 \\
\hline & 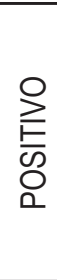 & 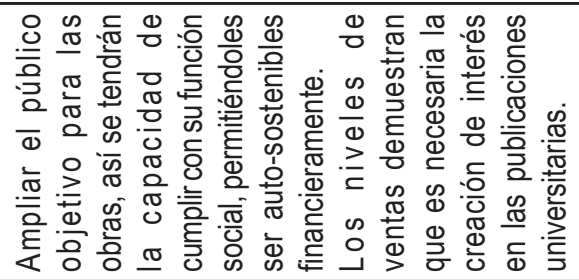 & 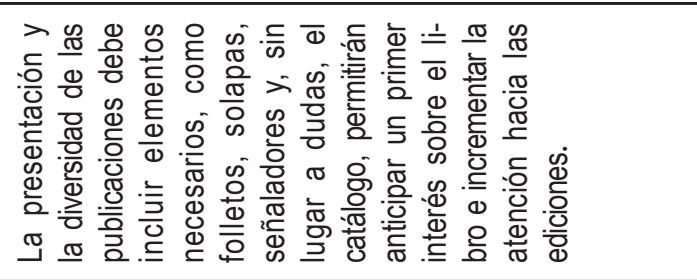 \\
\hline \multirow{2}{*}{ 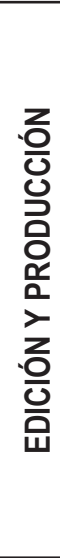 } & $\begin{array}{l}\stackrel{O}{Z} \\
\text { 离 } \\
\text { 㞱 }\end{array}$ & 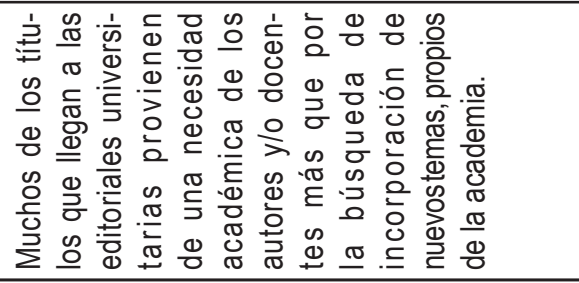 & 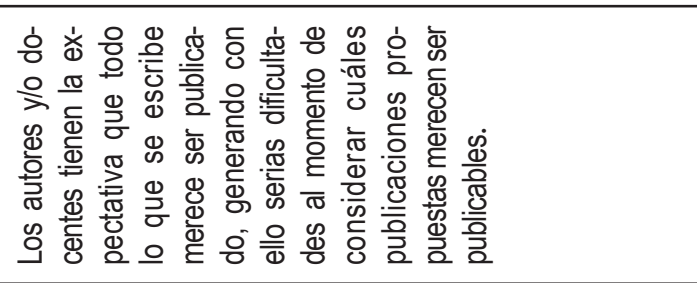 \\
\hline & 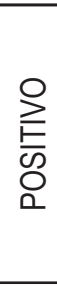 & 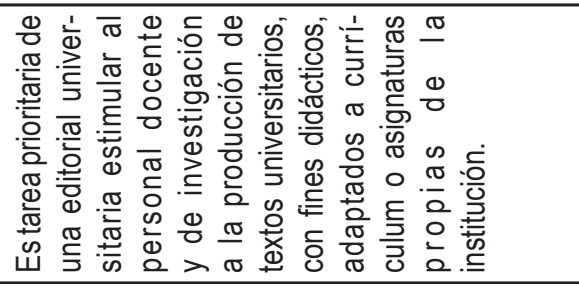 & 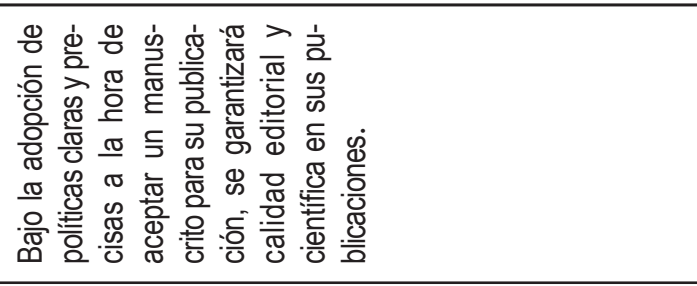 \\
\hline \multirow{2}{*}{ 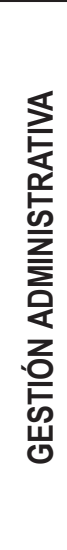 } & $\begin{array}{l}\stackrel{O}{Z} \\
\text { 离 } \\
\text { 岁 }\end{array}$ & 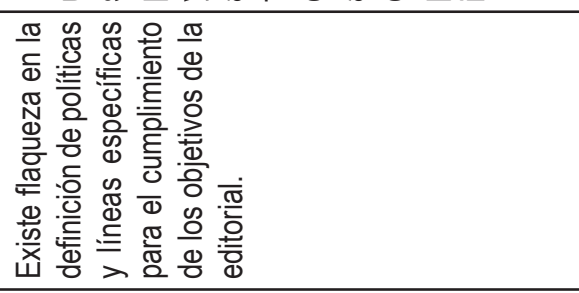 & 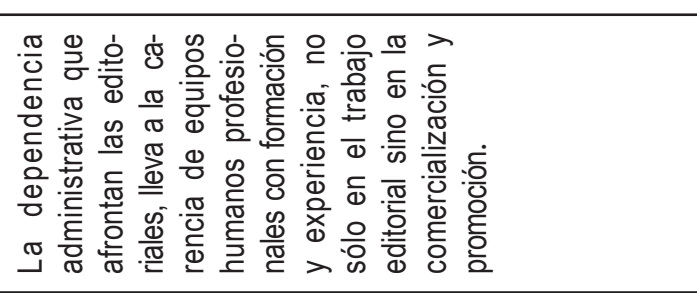 \\
\hline & 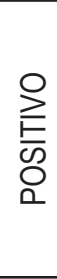 & 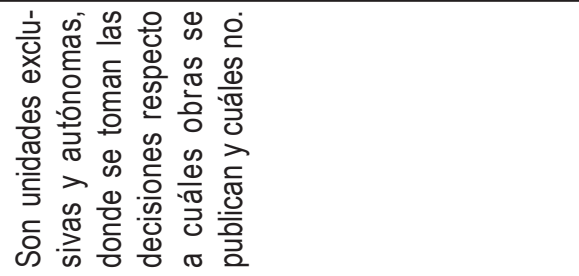 & 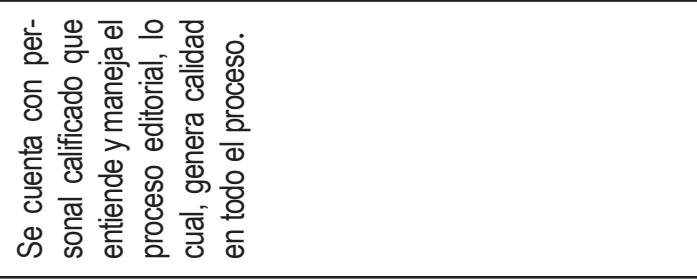 \\
\hline
\end{tabular}




\begin{tabular}{|c|c|}
\hline 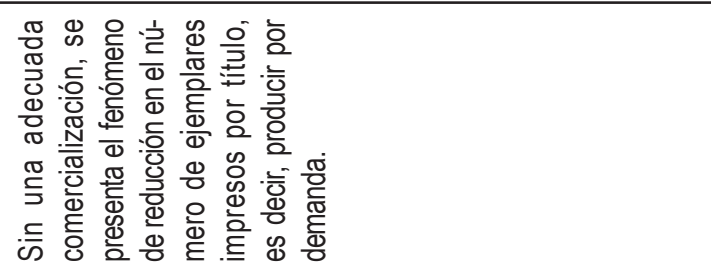 & \\
\hline 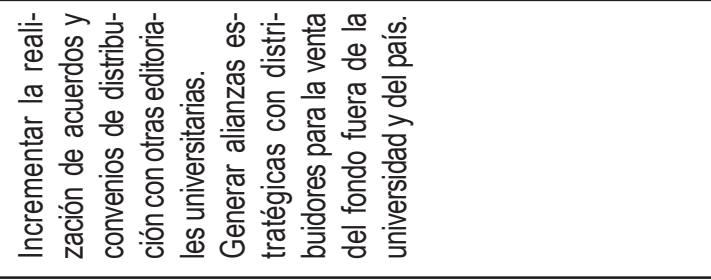 & 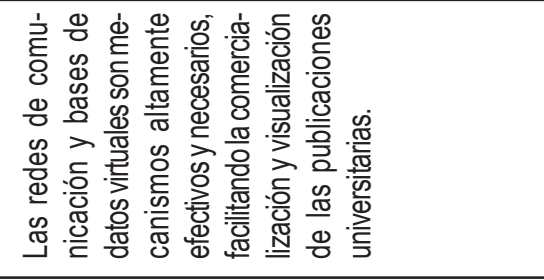 \\
\hline 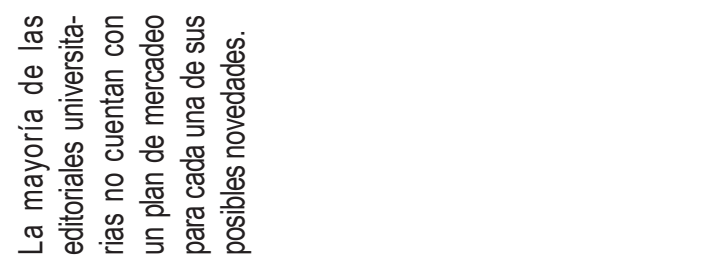 & 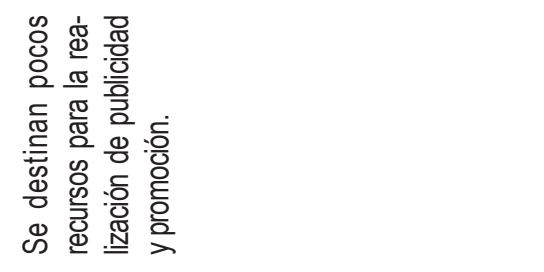 \\
\hline 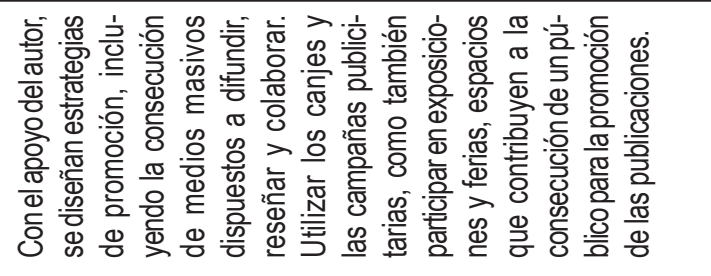 & 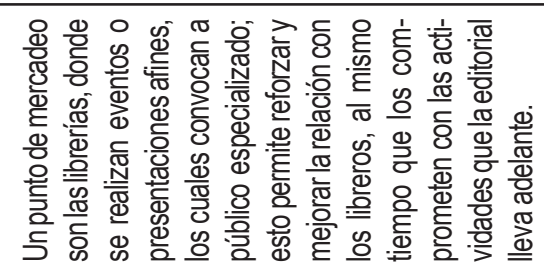 \\
\hline 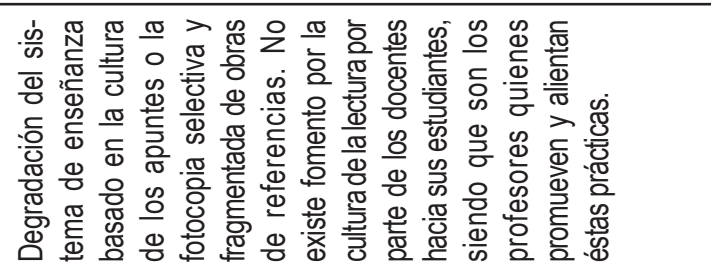 & 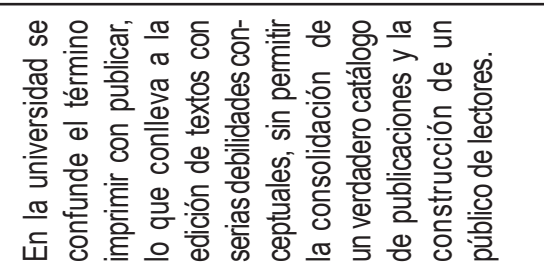 \\
\hline 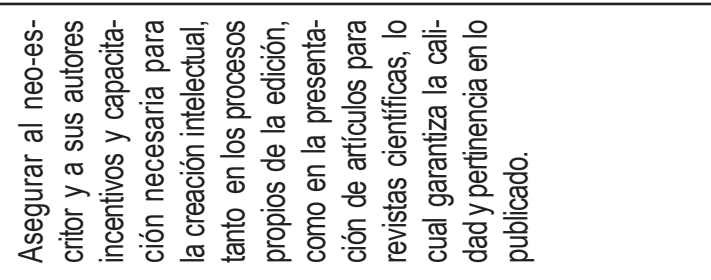 & 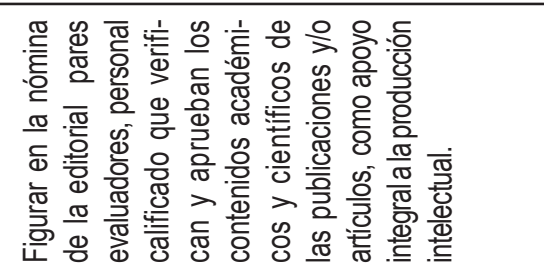 \\
\hline 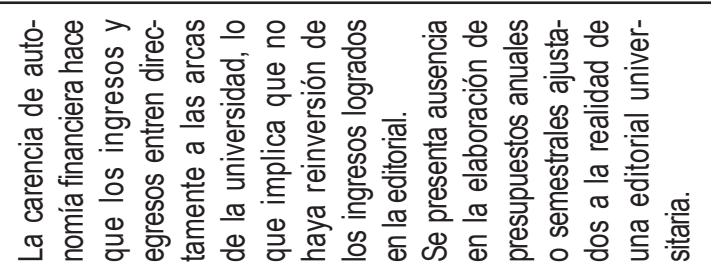 & 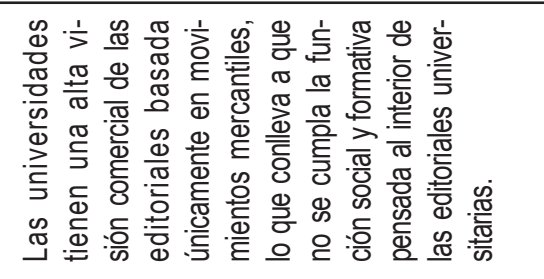 \\
\hline 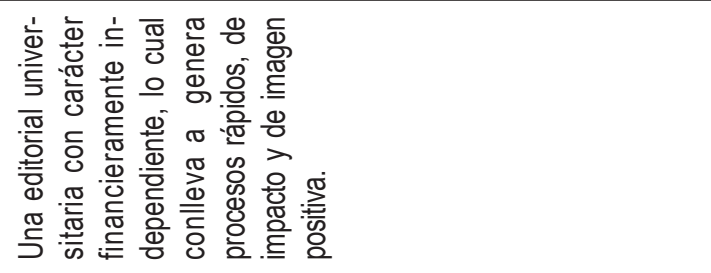 & 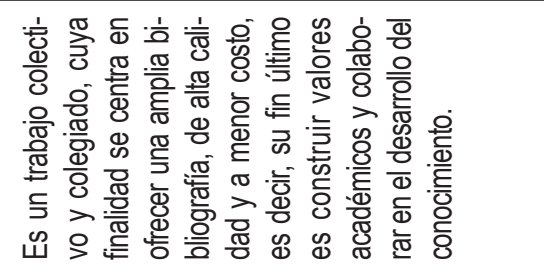 \\
\hline
\end{tabular}




\section{ESTADO ACTUAL DEL FONDO DE PUBLICACIONES DE LA U.D.C.A}

El Programa de Publicaciones de la U.D.C.A fue creado en el año 2000, bajo la resolución No. 109, del 2 de agosto, en el cual se reglamentó las actividades para el mismo y cumplir así con la responsabilidad que tiene ante la sociedad, dentro de sus funciones de docencia, de investigación y de extensión. El objetivo principal de crear e implementar el programa fue el de garantizar la edición, la promoción y la difusión de toda la producción intelectual, científica, artística y cultural creada por diferentes miembros de la comunidad universitaria. Una vez concebida esta idea, se estructuró el programa de publicaciones, con una Coordinación del programa y un Comité de Publicaciones fijándole a cada uno funciones y responsabilidades, propias del oficio editorial (Figura 1).

\section{ORGANIGRAMA (ANTES Y DESPUÉS)}

EDI-U.D.C.A

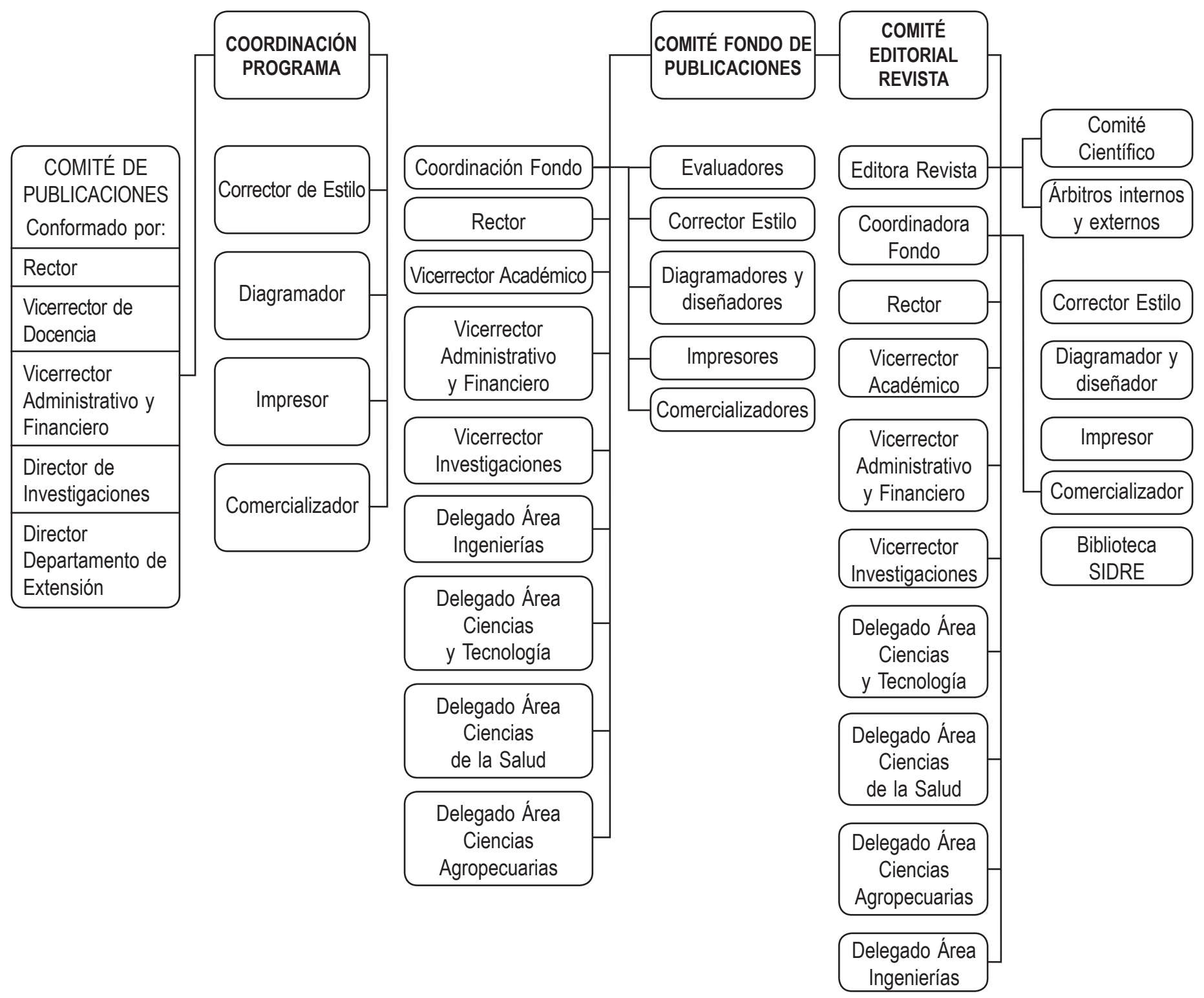

Figura 1. Estructura Orgánica del Programa y los órganos que garantizan la calidad de las publicaciones. 
Desde la creación del Programa de Publicaciones, antes EDI-U.D.C.A y actualmente Fondo de Publicaciones, pero especialmente en los últimos años, se puede decir que se ha afianzado y fortalecido la producción intelectual de la Universidad, a través de la consolidación de esta función. Se dio forma a las publicaciones, con la producción de libros, de manuales y, especialmente la edición de la Revista Científica, todos ellos emanados de la actividad investigativa, del ejercicio profesional y de la actividad universitaria.

El Fondo de Publicaciones de la U.D.C.A muestra su evolución en la forma de entender su gestión editorial, desde su óptica más global, que implica pensar, producir y difundir el libro como un bien cultural para el desarrollo de la academia y de la sociedad incluyendo el trabajo técnico de corrección de pruebas, de impresión y de comercialización. A través de su objetivo, ha propiciado una eficiente difusión social, con el cumplimiento de las siguientes funciones:

- Estímulo a la producción intelectual en la comunidad académica.

- Creación de conciencia, a nivel de las autoridades universitarias de la importancia de las editoriales universitarias.

- Apoyar las coediciones con editoriales universitarias y comerciales, con el objeto de reducir costos y mejorar los sistemas de distribución.

- Definición de criterios comunes para mejorar sus ediciones y sus estrategias para optimizar la distribución de sus publicaciones.

- Buscar el establecimiento de sistemas interuniversitarios de distribución y de mayor acceso a un mayor número de usuarios, a través de la participación en eventos feriales y el establecimiento de sus relaciones editoriales.

\section{Fortalezas del Fondo de Publicaciones:}

- Existencia de un Comité de Publicaciones y Comité de la Revista identificado como el órgano asesor que rige las políticas para editar obras de carácter académico, científico y cultural, lo cual garantiza la unidad formal y conceptual.

- Ubicación del Fondo en el organigrama de la Universidad, adscrito directamente a la Rectoría, cuya participación y apoyo en el establecimiento de las políticas editoriales es fundamental para el desarrollo de sus actividades.

- Edición de textos universitarios con temas especializados acorde con las áreas de conocimiento ofrecidas por la universidad: Medicina Veterinaria, Zootecnia, Ingeniería Agronómica, Ingeniería Geográfica y Ambiental y Medicina, entre otros, que son demandados por el público externo.

- Incremento en el número de títulos elaborados por los docentes, derivado de la política de asignar tiempo para la elaboración de futuras ediciones.

\section{Debilidades del Fondo de Publicaciones:}

- No se cuenta con el personal suficiente para esta función.

- Presupuestalmente, se depende de las decisiones de la Vicerrectoría Administrativa y Financiera, referente a montos destinados para su ejecución.

- La mayoría del material para publicar es pensado únicamente para los estudiantes y no para público externo.

- No existen políticas de comercialización para la producción editorial.

\section{Oportunidades del Fondo de Publicaciones:}

- Inicio de convenios de coedición con editoriales universitarias y/o comerciales.

- Implementación de líneas y productos para la edición de nuestras publicaciones, novedosas, impactantes y actuales.

- Creación de novedosos canales de distribución y/o comercialización.

\section{FONDO DE PUBLICACIONES U.D.C.A EN CIFRAS}

Con el fin de poder cuantificar y cualificar las ediciones en un Fondo de Publicaciones y, específicamente las de la U.D.C.A, se hace necesario definir y adoptar unas líneas editoriales y así clasificar su resultado, en lo que se refiere a cantidad de ejemplares editados, líneas y/o series con mayor movimiento y tipo de modalidad más solicitado, es decir, libro, revista, manual, cartilla y otro. Sin embargo, el Fondo a la fecha ha logrado categorizar su producción académica y científica, tal como se demuestra en la figura 2. 


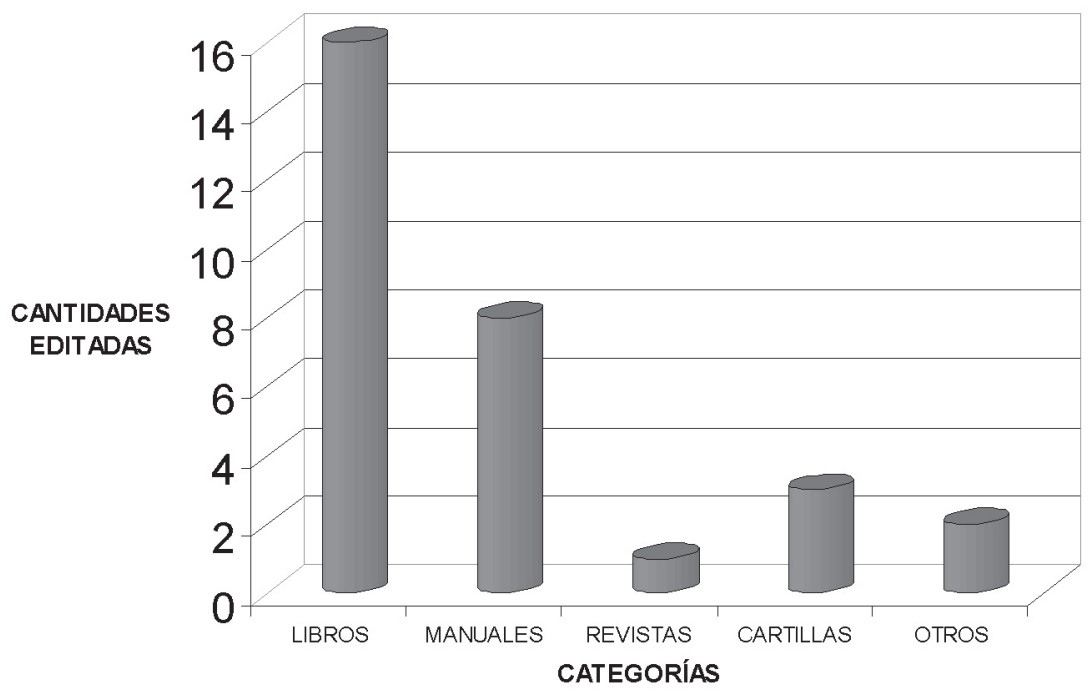

Figura 2. Tipos de publicaciones editados en el Fondo.

De las cifras consignadas en estas figuras, se puede deducir que el Fondo edita sus libros en las áreas académicas. Desde su creación, se han impreso más de 15.000 ejemplares y un total de 28 títulos. De la cantidad de ejemplares, un $78 \%$ ha sido vendidos o donados, indicando que el stock o bodegaje de libros es bajo.

El Comité de Publicaciones, en su sesión del primero de octubre de 2007 y registrada en Acta No. 038, aprobó un número representativo de manuscritos para ser editados, bajo la política de incrementar nuestra producción académica y científica, apoyando y avalando la creación intelectual de los docentes, mediante la asignación de tiempo en la planeación académica de cada programa, metodología e incentivo implementado a finales del año 2002 y que actualmente orientan y aprueban las decanaturas de las diferentes facultades (Figura 3).

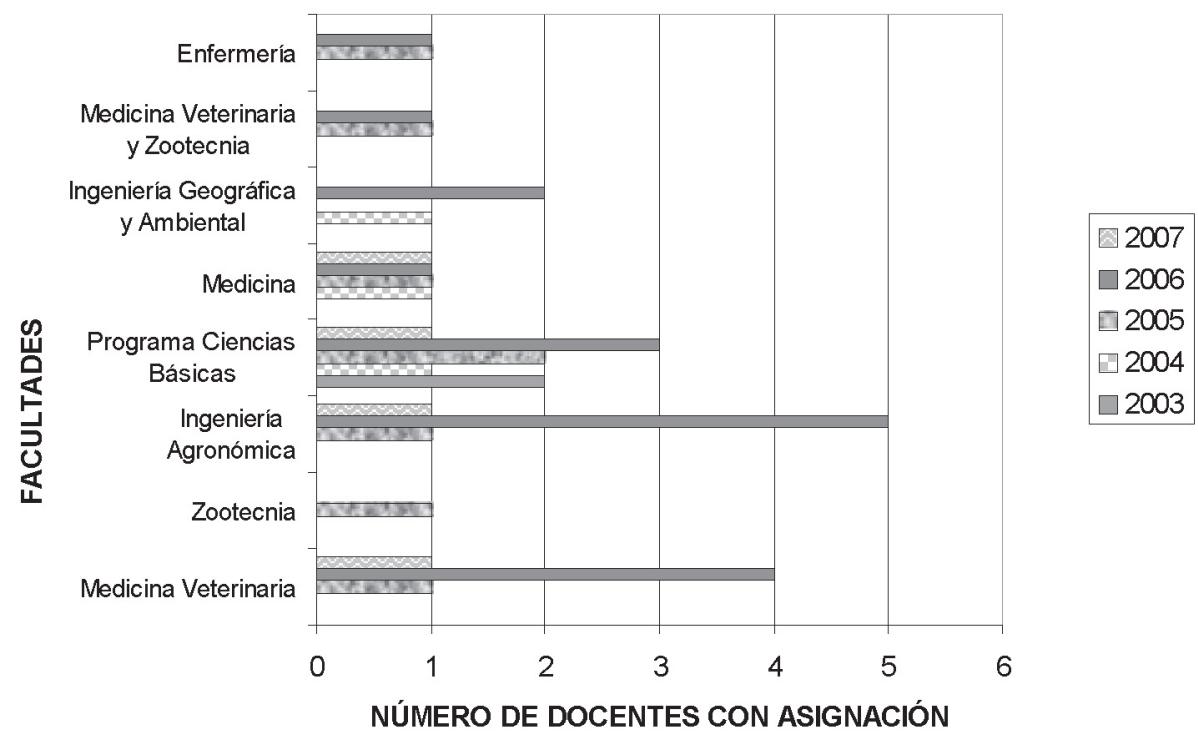

Figura 3. Número de docentes por programa académico con asignación semanal para la elaboración de publicaciones. 
Como complemento a este incentivo, los docentesautores tienen la ventaja, dentro de su política editorial, que la Universidad cubre el $100 \%$ los costos de la edición de su obra y todas sus fases del proceso.

Como parte de la responsabilidad del Fondo de Publicaciones con sus autores-docentes es el reconocimiento justo y acorde a su trabajo académico y de investigación, en la fase de derechos de autor; ello se refiere a brindarle un porcentaje, en libros y/o económico, sobre el tiraje, lo mismo que con la Revista Científica. Desde el segundo semestre del año 2003, se inició el proceso de firma y de registro de contratos de edición con cada uno de ellos, teniendo en cuenta todas las disposiciones legales y vigentes sobre la materia, garantizando de parte y parte confianza y vínculos más estrechos.

Como complemento a las gestiones propias del quehacer de una editorial universitaria, como función de difusión, el Fondo de Publicaciones asume los procesos de mercadeo y de comercialización, cuyo propósito es hacer visible y promover el avance de la ciencia, como también preservar y difundir el conocimiento. Sin un afán mercantilista y sin predominar el lucro, el Fondo ha buscado, entre muchas alternativas, hacer presencia en diferentes eventos académicos del libro, que le permitan su supervivencia y su desarrollo y volverse más eficiente y vigorosa. Como resultado de esta gestión, la U.D.C.A ha participado en las diferentes versiones de la Feria Internacional del Libro de Bogotá, con stand individual y conjuntamente con la Asociación de Editoriales Universitarias de Colombia ASEUC. Con gran éxito, en las instalaciones del Campus Universitario, logró el montaje de tres Ferias del Libro Universitario (2003, 2004 y 2005), con el firme propósito de instalar la cuarta versión, en el mes de mayo de 2008, en el marco de los 25 años de la Universidad.

Todas estas acciones de promoción y de difusión del conocimiento emanado de nuestros docentes de la U.D.C.A, ha traído consigo reconocimiento científico y académico tanto nacional como internacionalmente, debido a la oportunidad de comercializarnos en otros países, como Venezuela, México, Ecuador, Perú, Costa Rica y, recientemente, en España y Argentina; hemos logrado, también, realizar los primeros pasos en la coedición con editoriales comerciales. Es una responsabilidad que la estamos articulando satisfactoriamente, como lo han alcanzado otros grandes
Centros Editoriales Universitarios de Colombia y como lo reseña la Cámara Colombiana del Libro (2004), en los casos específicos como la Escuela Colombiana de Ingeniería, Universidad de Los Andes, Universidad de La Sabana, Politécnico Grancolombiano, Universidad de Medellín y otras más.

\section{PROPUESTA DE TRABAJO: UNA REALIDAD PARA NUESTRO FONDO}

La propuesta tiene como objetivo formular los criterios que promuevan un proceso de comunicación integral, permitiendo la consolidación de un sello y editorial universitaria, teniendo como referente la responsabilidad en la orientación, la búsqueda, la obtención, la evaluación y la selección de excelentes publicaciones y posterior difusión, que respondan a las verdaderas inquietudes de una comunidad educativa y social. Para su cumplimiento, se requiere de un trabajo colectivo y colegiado con las diferentes instancias y estamentos de la universidad. La constitución de una organización académico-administrativa debe abarcar alcances de responsabilidad que impulsará al Fondo de Publicaciones a un mejor escalafón.

\section{Gestión Administrativa:}

- Diseñar e implementar la política del Fondo de la U.D.C.A, coherente con el proyecto educativo institucional y con los proyectos de cada facultad.

- Plena identificación del quehacer editorial en el ámbito universitario y fuera de él, con el propósito de elevar el conocimiento.

- Determinar las líneas editoriales en Ciencias de la Salud, Arte, Medicina Veterinaria, Administración, Enfermería, Pedagogía, entre otras, con la participación de las facultades, que promueven la generación de conocimientos resultantes de la actividad académica, mediante la publicación y difusión de obras con valor científico, histórico, cultural, pedagógico, artístico o literario.

- Crear el Comité Académico para cada una de las facultades, asignándole responsabilidades y consolidar las funciones para cada uno de los miembros de Comité de Publicaciones, tanto a nivel académico como administrativo.

- Obtención de su plena autonomía administrativa y financiera, con el apoyo irrestricto de la rectoría y 
aquellas unidades que tengan que ver directamente con la labor del Fondo de Publicaciones.

\section{Edición y Producción}

- Crear y fomentar el uso del manual de publicaciones y su política editorial.

- Asesorar a los neoescritores y docentes-autores en los procesos de la escritura, de la redacción y presentación de artículos para revistas científicas y/o especializadas.

- Crear un mecanismo para estimular al personal docente y de investigación a la producción de textos universitarios, con fines académicos y también a la edición de obras literarias y de extensión del conocimiento, que contribuyan al rescate y afianzamiento de la cultura del país.

- Fortalecer las acciones de reconocimiento a los autores para generar sentido de pertenencia hacia la Institución y una identidad hacia el Fondo de Publicaciones, elaborando un proyecto de Derechos de Autor.

- Garantizar la calidad en los contenidos y en la presentación de sus publicaciones, como mecanismo para la futura comercialización y mercado y para el reconocimiento y obtención de una buena calificación académica.

- Formar un banco de evaluadoresy/o pares académicos, según su especialidad y/o conocimiento.

- Consolidar la imagen tanto institucional como del Fondo de Publicaciones, a través de sus ediciones novedosas, de impacto y de alto contenido.

\section{Mercadeo y Comercialización:}

- Institucionalizar el sello editorial.

- Seleccionar adecuadamente la presentación de nuestras publicaciones, en el tipo de formato, ya sea en físico o electrónico, teniendo en cuenta el impacto que tendrá la obra y su público primario y secundario.

- Fijar una visión sobre el proceso de mercado y comercialización, que oriente a los docentes en qué áreas o géneros se puede incursionar y que aporten a la sociedad y su circulación y/o visualización se de impacto.

- Fortalecer y definir los canales de distribución y comercialización, con el fin de diseñar y gestionar estrategias efectivas de mercadeo y promoción, para el desarrollo permanente de la labor del Fondo.

- Organizar y conformar la librería universitaria dentro del Campus Universitario, en una de sus sedes y con atención al público en general.

- Ampliar la gama de servicios editoriales y temas de publicación.

- Conformar un grupo de trabajo dedicado a la edición que incluya diseñadores, evaluadores, correctores de estilo y comercializadores, entre otros.

- Elaborar un catálogo con una serie de colecciones, para promover y divulgar la producción editorial. Esta herramienta se hace necesaria para llevar a diferentes eventos feriales, tanto a escala nacional como internacional.

- Crear una cultura institucional contra la fotocopia y la piratería, a través del Centro Colombiano de Derechos Reprográficos CEDER.

- Crear una política de participación en eventos feriales.

- Programar actividades académicas y culturales que tengan que ver con el mundo del libro: Conversatorios sobre Derechos de Autor, Talleres sobre Producción Editorial, dirigidos tanto para docentes, como para administrativos.

- Garantizar la calidad, la conveniencia y la pertinencia de las publicaciones.

\section{CONCLUSIONES}

Las editoriales universitarias en Colombia han evolucionado, adaptándose a los modos de comunicación científica y cultural determinados por las tecnologías de la información y los hábitos de los lectores. En la actualidad difunden, por medios impresos y electrónicos, el conocimiento generado en las universidades, contribuyendo a la transmisión del saber, la creación científica y el aprendizaje.

En su quehacer editorial, han arrojado resultados con estándares de calidad a través de sus publicaciones, aportaciones de sus docentes e investigadores, que las han llevado a incursionar en el ámbito de las editoriales universitarias más reconocidas a nivel nacional e internacional, jugando un papel importante en el desarrollo y afianzamiento en la excelencia de la educación superior, como oportunidad para la mejora de la enseñanza y el aprendizaje. 
El libro y la transmisión del conocimiento están en permanente cambio en la sociedad actual y es donde las editoriales universitarias colombianas han afrontado e integrado satisfactoriamente estas formas de publicación y difusión del saber tradicional y nuevo, desde el equilibrio entre los libros y los bytes, los formatos clásicos y los nuevos y el respeto del derecho de autor. Los fondos y las editoriales seguirán buscando herramientas estratégicas de promoción, de difusión, de venta, de atracción de lectores y disposición de contenidos para sus públicos, de acuerdo a las necesidades de autores y lectores.

El Fondo de Publicaciones de la U.D.C.A y las editoriales universitarias colombianas han potencializado la presencia del libro académico en Latinoamérica, en Europa y en Estados Unidos, utilizando todos los sistemas de distribución y comercialización existentes. La presencia de ellas en diferentes eventos y ferias del libro es el papel que juegan a diario, junto con sus docentes y bibliotecarios, para el impulso de la edición universitaria.

Tras siete años de existencia, el Fondo emprendió una nueva etapa de intensificación de sus actividades editoriales, de impulso a la edición universitaria, con la profundización en su presencia internacional, el desarrollo de nuevas estrategias de promoción. Nuestro Fondo de Publicaciones, no es ni será ajeno a la innovación, pues seguirá contribuyendo a la creación del conocimiento, fortaleciendo el desarrollo social y comprometiéndose con el aporte de editar libros con calidad científica y cultural.

\section{BIBLIOGRAFÍA}

ANÓNIMO. 2005. Términos de referencia para los estudios editoriales en América Latina. Disponible desde Internet en: www.iesalc.unesco.org.ve/programas/términos_referencia/tdreditorialesuniv.pdf (con acceso 27/10/06).

ANÓNIMO. s/f. Historia del libro. Disponible desde internet en: www.libreriasdecristal.com.mx. (con acceso 14/06/07).

CARDONA, T.C; SERRANO, J,J,; DELVASTO, G.A. 2007. Políticas Editoriales. Editorial Universidad Santiago de Cali, Colombia. 134p.
CAVALIERE, A. 2005. El libro impreso y el libro digital: estudio sobre los modos de producción editorial en el cambio de milenio. Universidad de Alicante. España Disponible desde Internet en: www.primeravistalibros/fichaLibro.jsp?idLibro $=1786$ (con acceso 05/08/07).

CÓRDOBA, J.; MARKEN, S.; VALBUENA, S. 2003. Balance preliminar de la actividad editorial universitaria en Colombia. En: Serie Documental. Estudios sobre la educación en Colombia. ASCUN- UNESCO-IESALC: Bogotá, Colombia. 112 p.

DE SAGASTIZÁBAL, L.; RUBIO, L.; 2004. Editoriales universitarias de América Latina y el Caribe. En: Las editoriales universitarias hacia el siglo XXI. Buenos Aires, Argentina. Capítulo 16. Disponible desde Internet en: www.iesalc.unesco.org.ve/pruebaobservatorio/informe rama eslat/capitulo xvi.pdf (con acceso 11/10/06).

DE SAGASTIZÁBAL, L. (2005). Presente y cambio en la edición universitaria. En: Memorias del I Foro de Edición Universitaria, Guadalajara: México. p. 34-50.

ESTADÍSTICAS DEL LIBRO EN COLOMBIA 2004. Cámara Colombiana del Libro, Bogotá. CD.

FURIÓ, A. 2005. El Futuro de la edición universitaria. En: Memorias del I Foro de Edición Universitaria". Altexto: Guadalajara, México. p. 9-24.

HERNÁNDEZ, H. 2005. La edición universitaria. Problemas, retos y propuestas de futuro. En: Memorias del I Foro de Edición Universitaria, Guadalajara: México. p.133-135.

POLO, M. 2005. Nuevas Estrategias de Edición Universitaria. En: Memorias del I Foro de Edición Universitaria, Guadalajara: México. p.25-26.

SICARD, A. 2005. Lineamientos para orientar la formulación de una política editorial de la Universidad Nacional de Colombia. Unibiblos. Colombia. Disponible desde Internet en: www.unibiblos.unal. edu.co/documentos/presentaciones/lineamientos_formulacion_politicaeditorial.nov2005ppt10 (con acceso 14/09/06). 
SIERRA, J.A. 2005. Marketing para editoriales universi- Recibido: noviembre 14 de 2006 tarias en el siglo XXI. DirectLibros. San José de Aceptado: octubre 30 de 2007 Costa Rica, Costa Rica. 260p. 